\title{
Clear Cell Myo-Melanomas of Ligamentum Teres Hepatis: A Case Report and Literature Review
}

\author{
Changcen Zhou',2, Yalin Zhang' \\ ${ }^{1}$ Graduate School, Hunan University of Traditional Chinese Medicine, Changsha, China \\ ${ }^{2}$ Department of Radiology, Changsha Central Hospital, Changsha, China \\ Email: 13507369716@163.com
}

How to cite this paper: Zhou, C.C. and Zhang, Y.L. (2021) Clear Cell Myo-Melanomas of Ligamentum Teres Hepatis: A Case Report and Literature Review. Open Journal of Clinical Diagnostics, 11, 93-99.

https://doi.org/10.4236/ojcd.2021.114007

Received: November 17, 2021

Accepted: December 21, 2021

Published: December 24, 2021

Copyright $\odot 2021$ by author(s) and Scientific Research Publishing Inc. This work is licensed under the Creative Commons Attribution International License (CC BY 4.0).

http://creativecommons.org/licenses/by/4.0/ (c) (i) Open Access

\begin{abstract}
Introduction: Clear Cell Myomelanocytic Tumor (CCMMT) of ligamentum teres hepatis is a pathological classification of Perivascular Epithelioid Cell tumor (PEComa), which is rare clinically and easy to misdiagnose. Objective: To report a case of a rare type of PEComa located in the ligamentum teres hepatis. Case Report: A 22-year-old Asian female was diagnosed with abdominal mass during physical examination in September 2018, and was admitted to the general surgery department of our hospital that month. She was diagnosed with abdominal mass, the nature of which remains to be determined is: teratoma, pheochromocytoma or ganglioma. At the time of admission, the patient had no symptoms or signs, and no other medical history. The patient was diagnosed with an abdominal mass by abdominal plain scan and enhanced CT, whose nature was to be determined: pheochromocytoma, paragangliomas or other mesenchymal tumors, or giant lymph node hyperplasia. The patient underwent abdominal mass resection and appendectomy without incident, without any complications at discharge, and there was no significant difference in follow-up. Conclusions: The clinical data, imaging features and pathological features of one patient diagnosed with CCMMT in our hospital were retrospectively analyzed, and the literature was reviewed in combination with the research progress of CCMMT, in order to improve the understanding and diagnostic accuracy of this disease.
\end{abstract}

\section{Keywords}

Clear Cell Myomelanoma, Ligamentum Teres Hepatis, Imaging Diagnosis, Case Reports 


\section{Introduction}

The ligamentum teres hepatis is a part of the ligament that forms the free margin of the liver falcine ligament. It is formed by the remnant of the embryonic left umbilical vein degenerated atresia, which connects the liver to the umbilical cord and divides the left side of the liver into medial and lateral parts [1]. In 2013, the World Health Organization (WHO) defined Perivascular Epithelioid Cell tumor (PEComa) as a new mesenchymal tumor type, which includes Clear Cell Myomelanocytic Tumor (CCMMT) of the reticular ligament or sickle ligament, and it was also included Lymphangioleio-Myomatosis (LAM), and Clear Cell "Sugar" Tumor of the lung (CCST); Angiomyolipoma (AML) and non-specific PEComa (PEComa-not Otherwise Specified, PEComa-NOS) [2] [3]. At present, there are limited literature reports on CCMMT of ligamentous teres hepatis, which is a very rare location and prone to misdiagnosis. Its diagnosis mainly depends on pathology, and its clinical features and imaging diagnosis need to be further studied. The case report and literature review of this case are as follows.

\section{A Case Report}

A 22-year-old female patient presented with abdominal mass for more than 10 days on physical examination. No abdominal pain, abdominal distension, diarrhea, fever, nausea, vomiting and other discomfort. Since the onset of the disease, the patient has a good mental sleep, normal appetite, normal urine and feces, and no significant change in weight. She was healthy in the past and had no abnormal personal and menstrual history. She got married at the age of 21 and had one son. Her spouse and son are still alive.

Physical examination on admission: body temperature: $36.6^{\circ} \mathrm{C}$, pulse: 96 beats/min, blood pressure: $122 / 22 \mathrm{mmHg}$. Specialized examination: flat abdomen, no varicose veins, soft abdominal muscles, no tenderness or rebound pain in the whole abdomen, negative ink sign, no palpable mass in the whole abdomen, no obvious buckling pain in the liver and kidney areas, negative movable dullness, normal bowel sounds, no metal sound or air passing water sound.

Laboratory examination: There were no obvious abnormalities in the three routine tests, liver and kidney function, coagulation function, the first four items of blood transfusion and fasting blood glucose; no tumor marker examination was performed; no obvious abnormalities were found in chest X-ray; normal ECG.

Imaging examination: The patient underwent plain and enhanced abdominal and pelvic CT examination in our hospital on September 3, 2018, which showed a soft tissue mass of about $32 \times 27 \mathrm{~mm}$ in size in the axial view of plain CT (Figure $1(a)$ ) in the right middle abdomen, with irregular shape, shallow lobulated shape, clear boundary, patchy and speckled calcification foci within. After axial enhancement (Figures 1(a)-(d)), the mass presented obvious uniform and continuous enhancement; sagittal (Figures 1(e)-(h)) showed unclear boundary between the mass and the ligamentum teres hepatis, resembling a cherry with a pedicle; 


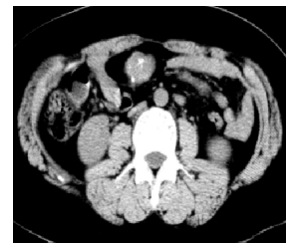

(a)

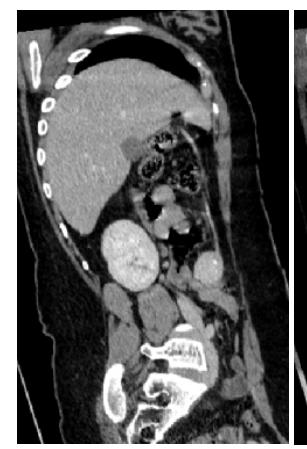

(e)

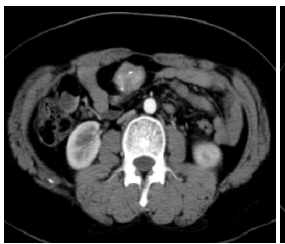

(b)

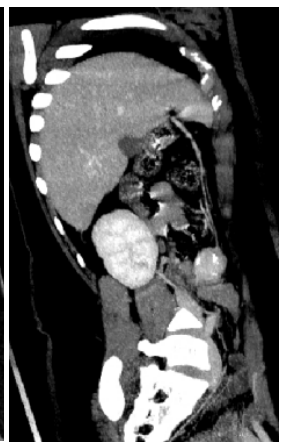

(f)

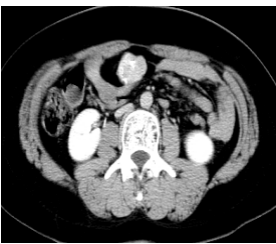

(c)

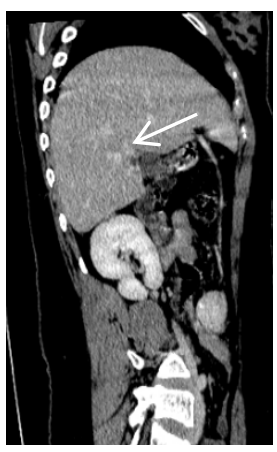

(g)

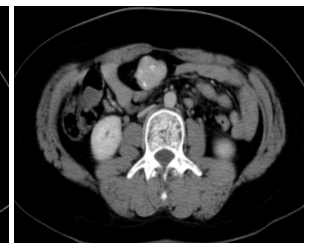

(d)

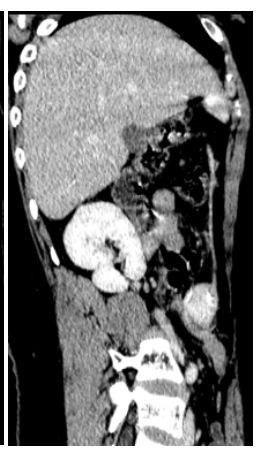

(h)

Figure 1. (a) Axial plain scan; (b) Arterial enhancement phase; (c) Venous enhancement phase; (d) Prolonged enhancement phase. Plain CT scan showed a soft tissue mass in the right middle abdomen, with a CT value of about $46 \mathrm{HU}, 138 \mathrm{HU}$ in arterial phase, $137 \mathrm{HU}$ in venous phase and $96 \mathrm{HU}$ in extended phase after enhancement. (e)-(h) is a delayed sagittal view with a soft tissue mass in the right middle abdomen and ligamentum teres ligamentum ligamentum punctate ( $\mathrm{f}$ white arrow).

no obvious signs of infiltration were observed in surrounding organs, and no obvious enlarged lymph nodes were observed in abdominal and pelvic cavity. Imaging diagnosis: its nature undetermined pheochromocytoma, paragangliomas or other mesenchymal tumors, or giant lymph node hyperplasia. Note: a small amount of pleural effusion on both sides.

Surgical findings: The patient was admitted to the general department of surgery of our hospital on September 17, 2018. The abdominal mass and appendectomy were performed under general anesthesia on the second day after admission. During the operation, there was no exudation in the abdominal cavity, and a $4 \times 5 \mathrm{~cm}$ mass on the round ligament $3 \mathrm{~cm}$ away from the incision was hard and mobile, infiltrating into the serosal layer, without adhesion to the surrounding greater omentum and bowel. The mass was completely removed $3 \mathrm{~cm}$ away from the edge of the mass by ultrasonic knife. The incision was inserted to open the fixator, and the abdominal cavity was explored. No palpable mass was found in the abdominal cavity, adhesion around the appendix, and the appendix was about $12 \mathrm{~cm}$ long.

Pathologic findings: One fatty mass, $6 \times 5 \times 3 \mathrm{~cm}$ in size, smooth grayish-brown surface, slightly lobulated/nodular, like a capsule, a nodule, $3.5 \times 2.5 \times 2.3 \mathrm{~cm}$ in size, grayish-white, bleeding, clear boundary, tough in quality, adjacent to the nodular adipoid tissue, grayish-yellow, soft, calcified foci formed inside. Immunohistochemistry: S100 (-), CgA (-), Syn (-), CD34 (vascular +), EMA (individual +), HMB45 (+), CD56 ( \pm ), Ki-67 (+1\%), AE1/AE3 (-), ALK (-), CD117 (-), 
TFE3 (-), Melan-A (-), Desmin (-), MyoD1 (small lesion +), H-caldesmon (+), CD68 (-), CD38 (plasma cell +), SMA (+), SOX10 (-), Vimentin (+) (Figure 2(a), Figure 2(b)). The pathology was consistent with a clear cell myopigmented tumor of the sickle ligament/round ligament.

The operation was successful, postoperative dressing was changed regularly, cefoxitin anti-infection, pantoprazole to protect the stomach, invert sugar electrolyte and compound potassium hydrogen phosphate fluid and other symptomatic supportive treatment. The patient was discharged 5 days after surgery, and the patient was in good condition during recent follow-up.

\section{Discussion}

CCMMT is most commonly found in ligamentum teres hepatis, followed by skin and bladder [4]. The etiology of this disease is still unclear, and some literatures suggest that it may occur simultaneously with the genetic disease tuberous sclerosis [5] [6]. The etiology of this disease is still unclear, and some literatures suggest that it may occur simultaneously with the genetic disease tuberous sclerosis [7]; Female patients were more than male; Patients with abdominal cavity and deep tissue were mostly younger. Occur in the skin of patients for the elderly [2]. The diagnosis of CCMMT mainly depends on pathology, immunohistochemistry and histology [8] [9], Immunohistochemistry was predominantly positive for HMB45 strong, SMA and actin were positive, and S-100 protein was partial positive [10]. In addition, CCMMT also has some special imaging features: 1) The diameter of the mass is less than $5 \mathrm{~cm}$, and hemorrhage and necrosis are uncommon; 2) The boundary of the capsule is clear and there is a fibrous pseudocapsule, but the tumor can also invade the capsule and the tissue outside the capsule; 3) The tumor is composed of spindle cells and "epithelioid cells", and the cytoplasm is abundant, so the plain CT scan usually shows equal or slightly lower density; 4) The tumor was rich in blood supply, obviously uneven enhancement in arterial phase after enhancement, and continuous enhancement in vein [4]. MRI of some cases also showed some characteristics, such as: 1) T1 and T2 showed high signal, which was similar to MRI of melanoma and might be

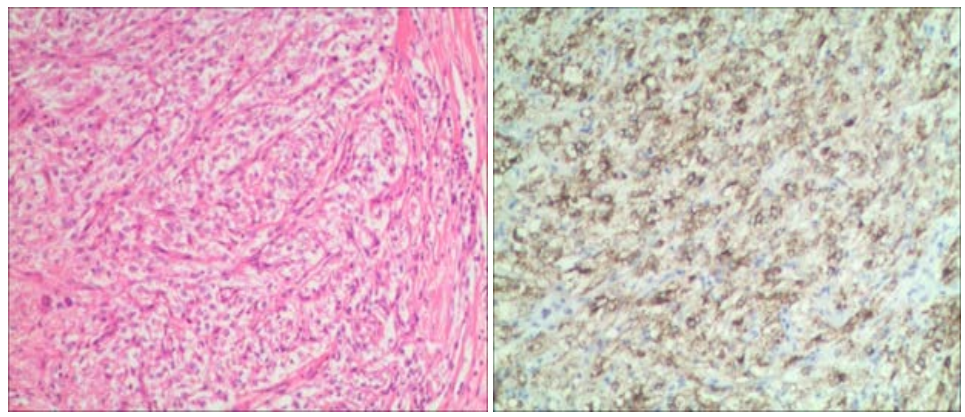

(a)

(b)

Figure 2. (a) and (b) are microscopic mass findings consistent with clear cell myo-pigmented neoplasms of the hepatic falcine ligament/round ligament. 
related to the content of melanin in the tumor [11];2) Limited diffusion on DWI sequence; 3) After enhancement, most of them showed obvious uniform enhancement [12]. In addition to the diagnosis of these manifestations, it is also necessary to differentiate tumors from other mesenchymal tissues in the abdominal cavity. It has to be recognized as Castleman's Disease (CD), of which men and women have the same diseases, most of them younger than 30 years old, and those diseases occur wherever lymph nodes still exist [13]. Pathologically, Castleman's disease is divided into clear cell type, plasma cell type and mixed type, among which the clear vascular type accounts for about $80 \%-90 \%$. In clinic, it can be divided into restricted type and diffuse type, in which the transparent vascular type is mostly confined type and the plasma cell type is mostly diffuse type [14]. The imaging manifestations of transparent cell Castleman's disease were similar to those of CCMMT, with the following characteristics: 1) single masses were mostly presented, with clear boundary and uniform density. CT enhanced significantly uniform enhancement was observed in arterial phase, and continuous enhancement was observed in portal vein phase and extended phase, and the enhancement degree was similar to that of aorta; 2) cystic degeneration, necrosis and calcification are rare, and calcification has its own characteristics, showing arc and branching; 3) All lesions showed slightly higher or equal signal on T1WI compared to muscle, higher or equal signal on T2WI, uniform enhancement after enhancement, and high signal on DWI [15]. In addition to identifying Ectopic Pheochromocytoma (EP), EP secrete plenty more catecholamine caused palpitations, symptoms such as headache, sweating, and high blood pressure, it has double the $10 \%$ rule, including $10 \%$ for bilateral sex, $10 \%$ for heterotopic, usually located in the abdominal aorta, renal hilum and mesenteric root and other parts [16], the imaging features of the tumors are as follows: 1) Most of the masses are oval, smaller ones have more uniform density and higher possibility of benign, while larger ones have more uneven density and higher possibility of malignant (diameter $>7 \mathrm{~cm}$ ); 2) The EP is Adrenal Pheochromocytoma (AP) potential malignant transformation; 3) The morphology was regular, but cystic degeneration and necrosis were common, and calcification was found in a few lesions; 4) Most of the mass on T2WI showed uneven and high signal intensity. Dynamic enhancement showed obvious enhancement of the lesion, and the lesion was slowly cleared over time [17].

\section{Conclusion}

Perivascular Epithelioid Cell tumor (PEComa) is not rare in the clinic, but PEComa located in the round ligamentum of the liver is very rare: its clinical characteristics are not obvious special, and the imaging examination and other mesenchymal tumor appearance are difficult to distinguish; its diagnosis and treatment of the gold standard is pathological biopsy and surgery. After reviewing this case, we should also identify this type of tumor when we encounter cases with similar imaging features in the clinic. 


\section{Patient Consent for Publication}

The patient consented for images or clinical information relating to his case to be reported in a medical publication.

\section{Conflicts of Interest}

The authors declare no conflicts of interest regarding the publication of this paper.

\section{References}

[1] Wang, H.T. (2013) Study on the Distribution and Function of Ligamentum Terule Endothelial Cells in Adult. Binzhou Medical College, Binzhou.

[2] Pan, C., Chen, S.H., Hong, D.X., et al. (2007) Clinicopathological Analysis of Clear Cell Myomelanocytoma in Lung. Fujian Journal of Medicine, 2-4.

[3] Wang, F.H., Cheng, L., Zhang, P.H., et al. (2021) Uterine Perivascular Epithelioid Cell Tumor with Bone Metastasis: A Case Report and Literature Review. Progress in Modern Obstetrics and Gynecology, 879-880.

[4] Wang, Z.S., Xu, L., Ma, L., Song, M.-Q., Wu, L.-Q. and Zhou, X. (2015) Hepatic Falciform Ligament Clear Cell Myomelanocytic Tumor: A Case Report and a Comprehensive Review of the Literature on Perivascular Epithelioid Cell Tumors. BMC Cancer, 15, Article No. 1004. https://doi.org/10.1186/s12885-015-1992-4

[5] Thway, K. and Cyril, F. (2015) PEComa: Morphology and Genetics of a Complex Tumor Family. Annals of Diagnostic Pathology, 19, 359-368. https://doi.org/10.1016/j.anndiagpath.2015.06.003

[6] Tan, Y., Zhang, H. and Wang, X.C. (2014) Clear Cell Myomelanocytic Tumor of the Falciform Ligament/Ligamentum Teres. Indian Journal of Pathology and Microbiology, 57, 453-455. https://doi.org/10.4103/0377-4929.138766

[7] Stuart-Lauren, N., Tipton-Russell, G., DeWall-Michael, R., Parker, D.C., Stelton, C.D., Morrison, A.O., Coleman, L.W., Fosko, S.W., Vidal, C.I., Hurley, M.Y., Deeken, A.H. and Gardner, J.M. (2017) Primary Cutaneous Perivascular Epithelioid Cell Tumor (PEComa): Five New Cases and Review of the Literature. Journal of Cutaneous Pathology, 44, 713-721. https://doi.org/10.1111/cup.12972

[8] Folpe, A.L., Mentzel, T., Lehr, H.A., Fisher, C., Balzer, B.L. and Weiss, S.W. (2005) Perivascular Epithelioid Cell Neoplasms of Soft Tissue and Gynecologic Origin: A Clinicopathologic Study of 26 Cases and Review of the Literature. The American Journal of Surgical Pathology, 29, 1558-1575. https://doi.org/10.1097/01.pas.0000173232.22117.37

[9] Liu, C.H., Chao, W.T., Lin, S.C., Lau, H.-Y., Wu, H.-H. and Wang, P.-H. (2019) Malignant Perivascular Epithelioid Cell Tumor in the Female Genital Tract: Preferred Reporting Items for Systematic Reviews and Meta-Analyses. Medicine, 98, e14072. https://doi.org/10.1097/MD.0000000000014072

[10] Xing, X.W., Yi, G.Z., Wang, X.Q., et al. (2001) Clear Cell Mymelanocytoma of the Round Ligament of the Liver. Journal of Clinical and Experimental Pathology, 219222.

[11] Martignoni, G., Maurizio, P., Claudia, Z., Brunelli, M., Segala, D., Zamboni, G. and Bonetti, F. (2015) PEComas of the Kidney and of the Genitourinary Tract. Seminars in Diagnostic Pathology, 32, 140-159. https://doi.org/10.1053/j.semdp.2015.02.006

[12] Zheng, Y.F., Zheng, H.L., Wang, S., et al. (2021) Uterine Malignant Perivascular Epithelioid Cell Tumor: A Case Report and Literature Review. Chinese General Prac- 
tice, 24, 2364-2367.

[13] Liu, N.J., Liu, X.Q., Zhang, H.W., et al. (2015) Multislice Spiral CT Diagnosis of Small Omental Giant Lymphocyte Hyperplasia: A Case Report. Chinese Journal of Clinical Medical Imaging, 26, 73-75.

[14] Carrion, D.M., Alvarez-Maestro, M., Gómez-Rivas, J., Brygadyr, Y., García-Fernandez, E. and Martínez-Piñeiro, L. (2019) Challenging Diagnosis of a Solitary Retroperitoneal Mass: A Case Report of Castleman's Disease and Review of the Literature. Urologia Internationalis, 103, 245-248. https://doi.org/10.1159/000493511

[15] Zhao, S., Wan, Y., Huang, Z., Song, B. and Yu, J.Q. (2019) Imaging and Clinical Features of Castleman Disease. Cancer Imaging, 19, Article No. 53.

https://doi.org/10.1186/s40644-019-0238-0

[16] Jiang, C., Zhao, J., Sun, L., et al. (2020) A Case Report of Rare Ectopic Pheochromocytoma Adjacent to Pancreas. Medicine, 99, e20858. https://doi.org/10.1097/MD.0000000000020858

[17] Zhu, Z., Zhao, X., Dai, J., et al. (2015) CT and MR Findings of Retroperitoneal Ectopic Pheochromocytoma. Chinese Journal of Oncology, 37, 181-185. 